\title{
FINITE ELEMENT ANALYSIS OF ELASTOMERIC NETWORKS WITH FOCUS ON EFFECTS OF POROSITY
}

\author{
Mohammad Hossein Moshaei ${ }^{1 *}$, Payman Sharifi AbDaR $^{2}$, \\ MOHAMMADREZA AZMOODEH ${ }^{3}$ \\ ${ }^{1}$ Department of Mechanical Engineering, Ohio University, Athens, Ohio, US \\ ${ }^{2}$ Department of Chemical and Biomolecular Engineering, Ohio University, \\ Athens, Ohio, US \\ ${ }^{3}$ Department of Mechanical Engineering, University of Tehran, Tehran, Iran
}

[Received: 05 July 2019. Accepted: 21 October 2019]

doi: 10.7546/JTAM.50.20.01.06

\begin{abstract}
One of the common assumptions in analysis of polymer network on micromechanical level is the non-porosity of the polymeric media, but it is merely a simplifying assumption. This study dismissed this assumption and studies the polymeric networks with $10 \%$ and $20 \%$ porosity and compares them with a polymer media without porosity. To model the porosity in the polymer network, a random morphology is employed and using ABAQUS software, nonlinear finite element analysis is performed to study the structure of the porous polymer network. Comparing the networks with $10 \%$ and $20 \%$ porosity with the non-porous network shows the significant effect of porosity in mechanical behavior of the polymer network and how it can increase the maximum von-Mises stress remarkably.
\end{abstract}

KEY WORDS: elastomers, finite element method, polymer network, porous media.

\section{INTRODUCTION}

Porous materials have been one of the exciting subjects in physics and chemistry since the discovery of the zeolites and their application in the industry. From physical point of view, porosity is used as a key parameter in designing materials in order to modify their properties and improve their performance [1]. The area of porous materials has experienced a revolutionary growth during former decades. Of various porous materials, porous polymers such as metal-organic frameworks (MOFs), crystalline covalent-organic frameworks (COFs) and amorphous porous organic polymers (POPs) have been well developed and intrigued much attention as they not only possess the benefits of high surface area and well-designed porosity like conventional porous materials such as zeolites or activated carbons, but also provide the advantages of polymeric materials e.g. corrosion resistivity. Porous polymers are low

\footnotetext{
${ }^{*}$ Corresponding author e-mail: mm733315@ohio.edu
} 
weigh, chemically and thermally stable, easy processable materials. In addition, they are qualified to introduce chemical functionalities into the materials' porous framework by bottom-up or post-synthetic modification approach $[2,3]$. These remarkable features make porous polymers as promising candidates for applications in catalysis and photocatalysis [4,5], gas separation [6], water and wastewater treatment [7], energy storage [8], drug delivery and tissue engineering [1,9].

Elastomers are type of polymers with unique properties such as high elasticity, large extensibility, good formability, and low cost. The polymeric network of elastomers formed by the connection of long chain molecules results in high elasticity which enable them to be applied in different areas [10] including automotive industry [11], electronic manufacturing [12], magnetorheological [13], and biomedical devices [14,15], and as a consequence the necessity of the study on the properties of elastomers has been arisen. Of more importance is the analysis of mechanical characteristics because elastomers are mostly undergone deformation. Effects of different parameters on the mechanical behavior of elastomers have been investigated by several researchers $[11,16]$. For instance, Tehrani et al. studied the effect of chain length distribution and polydispersity on the strength of polymeric networks and concluded that the mechanical strength of elastomeric networks has been notably influenced by the distribution of strands length $[11,17,18]$. However, these valuable researches ignore the significant effect of porosity by assuming a non-porous media for elastomers. On the other hand, considering porosity factor could be a huge enhancement in this area as porous elastomers are emerging type of novel materials, which have been increasingly used in different areas of biomedical application [14, 19]. Owing to these applications of porous elastomers, fluid-structure interaction (FSI) analysis, in the presence of porous elastomers as the solid structure, is critically required. While there has been conducted numerous studies over numerical analysis and CFD methods and its applications [20,21], investigating the mechanical properties and finite element analysis of porous elastomers has been barely touched [22]. One of the most useful techniques in finite element analysis, which have been applied credibly to study the mechanical behavior of elastomers and polymeric networks, is asymptotic expansion homogenization (AEH) method [23,24]. This technique is a rigorous and well-known approach to predict the properties of material using periodic microstructure [25].

The objective of this paper is to develop and implement a methodology for creating morphologically realistic heterogeneous random porous microstructures over the entire volume fraction range, and subsequently analyze their statistical and homogenized material properties in an effort to extract valuable insight into the behavior of realistic porous elastomer. To obtain that goal, the asymptotic expansion homogenization (AEH) method is used in conjunction with multiscale analysis to obtain the 
stresses at the microscopic and macroscopic levels.

\section{Methodology}

In this model, the random morphology description function (RMDF) is used to develop random microstructures, which can have different porosity.

$$
f(y)=\sum_{i=1}^{N} c_{i} \exp \left\{-\left[\frac{\left(y_{1}-y_{1}^{i}\right)+\left(y_{2}-y_{2}^{i}\right)}{w_{i}^{2}}\right]\right\},
$$

where $y^{i}$ and $c_{i}$ are random coordinates and random coefficient, respectively. $N$ in equation (1) is the number of random functions. Also:

$$
w_{i}=\frac{1}{\sqrt{N}} .
$$

This equation is consisted of two-dimensional Gaussian functions, which creates a realistic random function [26]. Using Eq. (1) with different cutoff values, random porous media with different porosity are modeled as presented in Fig. 1.

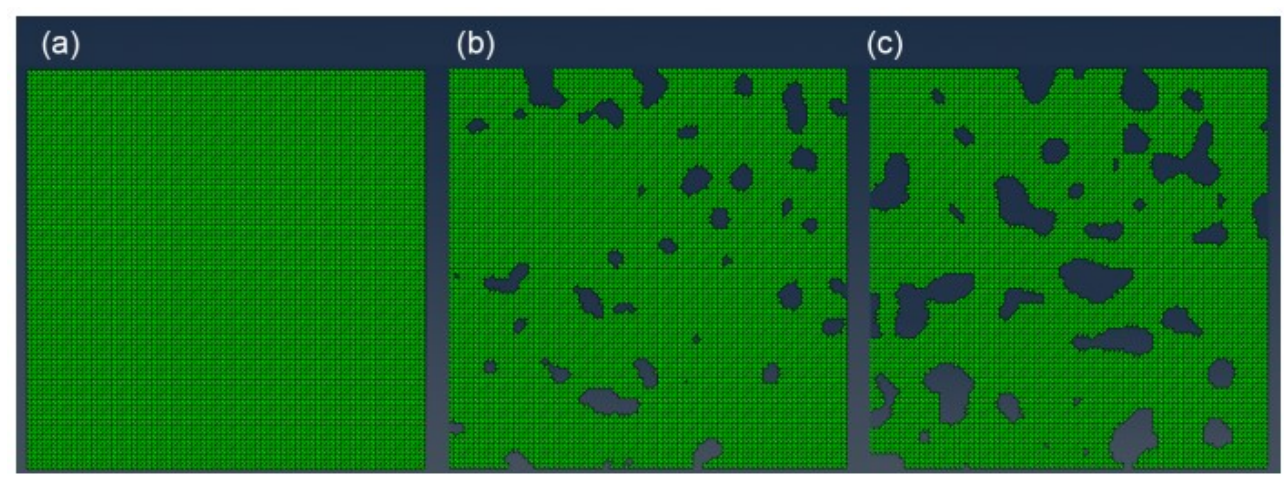

Fig. 1. (a) Nonporous media; (b) porous media with $10 \%$ porosity; and (c) porous media with $20 \%$ porosity.

Different phenomenological and statistical models $[27,28]$ have been developed to represent mechanical response of the polymer networks. Recently, Tehrani-Sarvestani model introduced new framework to study the mechanical behavior and failure in polymer networks $[11,17,18,29]$. This study employs Neo-Hookean model for polymer networks. The strain energy density function for a compressible Neo-Hookean material is given by $[11,17]$ :

$$
\psi(\lambda)=\mu \int_{0}^{2 \pi} \int_{0}^{\pi} \int_{j_{\min }(\lambda)}^{\infty} P(j) w(\lambda, j) \sin \theta_{0} d j d \theta_{0} d \phi_{0} .
$$


With $\mu=\frac{\sum n_{j}}{4 \pi V}$ where, $\sum n_{j}$ is the total number of existing strands and $V$ is the occupying volume. $\lambda$ is macroscopic stretch, $j$ is the length of the strands, and $w(\lambda, j)$ is the free energy of each strand. $j_{\min }$ is the length of the shortest strand and defined as [17]:

$$
j_{\min }(\lambda)=\frac{\lambda^{2}\left(\theta_{0}, \phi_{0}\right)}{\zeta}
$$

where $\theta_{0}$ and $\phi_{0}$ are the coordinates in the reference configuration and $\zeta$ is:

$$
\frac{1}{\zeta}=\frac{3(3+\sqrt{4 \gamma+9})}{2 \gamma^{2}}, \quad \gamma=\frac{\alpha a U_{0}}{2 k_{B} T},
$$

where $a$ is the characteristic length for one statistical segment, $U_{0}$ is the dissociation energy, and $\alpha$ is a constant for the bond elasticity.

In Eq. (6) $P(j)$ is the probability distribution of having a strand with $j$ statistical segments, as Tehrani derived in $[11,28]$ :

$$
P(j)=\frac{1}{\bar{j}} \exp \left(-\frac{j}{\bar{j}}\right) .
$$

Here $\bar{j}$ is the reciprocal of average strand length.

For nonlinear problems three stress measures are usually considered: the Kirchhoff stress, $\tau$, the Cauchy stress, $\sigma$, and the second Piola-Kirchhoff stress, $\boldsymbol{S}$. Considering strain energy to be function of the deformation gradient tensor $\psi=\psi(\boldsymbol{F})$ and $J$ to be the Jacobian of the deformation, the three measures of stress can be derived as [30]:

$$
\boldsymbol{S}=2 \frac{\partial \psi}{\partial \boldsymbol{C}}, \quad \boldsymbol{\sigma}=\frac{2}{J} \boldsymbol{F} \frac{\partial \psi}{\partial \boldsymbol{C}} \boldsymbol{F}^{T}, \quad \boldsymbol{\tau}=J \sigma,
$$

where $C$ the right Cauchy-Green tensor. It should be mentioned that the second PiolaKirchhoff stress is associated with total Lagrangian formulation while the other two are associated with the updated Lagrangian formulation [30,31]. For this study, the updated Lagrangian formulation in ABAQUS is adopted.

For user defined material models in finite element, based on different stress rates, different tangent modulus can be obtained, so it is important to implement an accurate objective rate of a stress tensor. Following Miehe [30,32], the convected rate of the Kirchhoff $\boldsymbol{L}_{\boldsymbol{v}} \boldsymbol{\tau}$ is:

$$
\boldsymbol{L}_{v} \boldsymbol{\tau}=\dot{\boldsymbol{\tau}}-\boldsymbol{L} \boldsymbol{\tau}-\boldsymbol{\tau} \boldsymbol{L}^{T}=\bar{C}^{\tau c}: D
$$


Here, $\bar{C}^{\tau c}$ is the tangent modulus tensor [30,32], $\boldsymbol{D}$ is the rate of deformation tensor, and $L$ is spatial velocity gradient. For ABAQUS continuum elements, based on Sun et al. [30], Jaumann rate of the Kirchhoff stress $\begin{aligned} & \nabla \\ & \tau\end{aligned}$ can be expressed as:

$$
\underset{\tau}{\nabla}=\dot{\tau}-W \tau-\tau W^{T}=\bar{C}^{\tau J}: D
$$

Here $W$ is spin tensor and $\bar{C}^{\tau J}$ is tangent modulus tensor for the Jaumann rate of the Kirchhoff stress:

$$
\bar{C}_{i j k l}^{\tau J}=\bar{C}_{i j k l}^{\tau c}+\delta_{i k} \tau_{j l}+\delta_{j l} \tau_{i k},
$$

where $\delta_{i j}$ is the Kronecker delta.

Following Miehe [32] a numerical approximation for tangent modulus is derived. The linearized form of Eq. (9) is:

$$
\Delta \tau-\Delta W \tau-\tau \Delta W^{T}=\bar{C}_{i j k l}^{\tau J}: \Delta D,
$$

where:

$$
\begin{aligned}
& \Delta \boldsymbol{W}=0.5\left(\Delta \boldsymbol{F} \boldsymbol{F}^{-1}-\left(\Delta \boldsymbol{F} \boldsymbol{F}^{-1}\right)^{T}\right), \\
& \Delta \boldsymbol{D}=0.5\left(\Delta \boldsymbol{F} \boldsymbol{F}^{-1}+\left(\Delta \boldsymbol{F} \boldsymbol{F}^{-1}\right)^{T}\right) .
\end{aligned}
$$

To find the estimate of the components of $\bar{C}^{\tau J}$, a small perturbation is required, so following Miehe [30], [32] one can obtain:

$$
\begin{aligned}
\Delta W^{(i j)} & =0, \\
\Delta D^{(i j)} & =\frac{\varepsilon}{2}\left(e_{i} \otimes e_{j}+e_{j} \otimes e_{i}\right),
\end{aligned}
$$

where $\varepsilon$ is a small perturbation. So, the perturbed deformation gradient is:

$$
\hat{\boldsymbol{F}}^{(i j)}=\boldsymbol{F}+\Delta \boldsymbol{F}^{(i j)} \text {. }
$$

So, the difference between perturbed and unperturbed Kirchhoff stress is:

$$
\Delta \tau \approx \boldsymbol{\tau}\left(\hat{\boldsymbol{F}}^{(i j)}\right)-\boldsymbol{\tau}(\boldsymbol{F})
$$

Then the numerical approximation of the tangent moduli is,

$$
\bar{C}^{\tau J(i j)} \approx \frac{1}{\varepsilon}\left[\boldsymbol{\tau}\left(\hat{\boldsymbol{F}}^{(i j)}\right)-\boldsymbol{\tau}(\boldsymbol{F})\right] .
$$


The material Jacobian in ABAQUS is:

$$
\bar{C}^{M J}=\frac{1}{J} \bar{C}^{\tau J}
$$

Inserting Eq. (19) in Eq. (18) the material Jacobian can be written as:

$$
\bar{C}^{M J(i j)} \approx \frac{1}{J \varepsilon}\left[\boldsymbol{\tau}\left(\hat{\boldsymbol{F}}^{(i j)}\right)-\boldsymbol{\tau}(\boldsymbol{F})\right] .
$$

Table 1 indicated the input mechanical properties of the polymer network in this ABAQUS simulation. The size of the specimen is $100 \mathrm{~mm} \times 100 \mathrm{~mm}$ and 6-node modified quadratic plane stress triangle elements (CPS6M) were used for the model to reduce mesh density without affecting solution accuracy.

Table 1. Material constants for Neo-Hookean model of polymer network in ABAQUS [33]

\begin{tabular}{cc}
\hline \hline Parameter & Value \\
\hline$C_{10^{*}}$ & $0.2587(\mathrm{MPa})$ \\
$D_{1}{ }^{* *}$ & $1.5825 e^{-3}$ \\
\hline \hline
\end{tabular}

${ }^{*}$ Neo-Hookean material constant for shear behavior

** Neo-Hookean material constant for bulk compressibility control

Figure 2 shows the schematic boundary condition of the finite element model. The implemented model is subject to a biaxial deformation control loading.

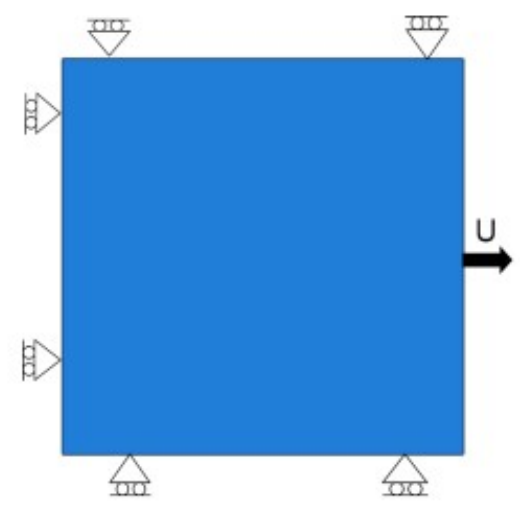

Fig. 2. Schematic boundary condition of finite element model. 


\section{RESULTS AND Discussions}

The model has been generated for heterogenous random porous polymer networks and the mechanical behavior of homogenized polymer networks have been obtained. Random porous media with $5 \%, 10 \%, 15 \%$, and $20 \%$ porosity have been generated and for each porosity percentage the simulation was repeated 10 times to check the validity and repeatability of each simulation. The average of maximum deformation and von-Mises stress are presented in Table 2. For simplicity and a better presentation, the results for the total deformations and von-Mises stresses are shown in Fig. 3, 4 and 5 for no porosity, $10 \%$, and $20 \%$ porosity.

Table 2. Average maximum deformation and von-Mises stress for different porosity percentage

\begin{tabular}{ccc}
\hline \hline $\begin{array}{c}\text { Porosity } \\
\text { percentage }\end{array}$ & $\begin{array}{c}\text { Average maximum } \\
\text { deformation }\end{array}$ & $\begin{array}{c}\text { Average maximum } \\
\text { von-Mises stress }\end{array}$ \\
\hline 0 & 0.0019 & $7.53(\mathrm{MPa})$ \\
5 & 0.572 & $20.93(\mathrm{MPa})$ \\
10 & 0.738 & $21.72(\mathrm{MPa})$ \\
15 & 1.18 & $22.08(\mathrm{MPa})$ \\
20 & 1.46 & $22.82(\mathrm{MPa})$ \\
\hline \hline
\end{tabular}

The average maximum von-Mises stresses in porous media with $10 \%$ and $20 \%$ are respectively $21.72 \mathrm{MPa}$ and $22.82 \mathrm{MPa}$, which are much higher than the average maximum von-Mises stress for the non-porous media, which is $7.53 \mathrm{MPa}$. According to the figures, stress contours demonstrate that not only the maximum von-Mises stress value is greatly increased in comparison with the non-porous media, but also with the increase in the porosity more area is subjected to higher stress. This means that increase in the porosity increases the chances for the rupture of polymer strands and ultimately, the failure of the polymer network. Owing to the geometry of the porous media, stress contours show severe von-Mises stress value in some specific regions like between two holes. These regions are readily susceptible to failure due to the stress value.

A finite element framework has been set up randomly and heterogeneously in order to relate the fraction of porosity and the mechanical behavior of porous polymer network. In this fashion, finite element method has been successfully applied to establish mechanical properties of the heterogeneous random porous polymer network. Initial simulation results have manifested that with increasing the porosity of media, the maximum von-Mises stress is simultaneously raised. This conclusion in drawn based on the results for $5 \%, 10 \%, 15 \%$, and $20 \%$ porosity. Furthermore, the results show that in the presence of the porosity in polymer networks, it is necessary to carry out structural analysis to map the stress field and predict failure behavior. 

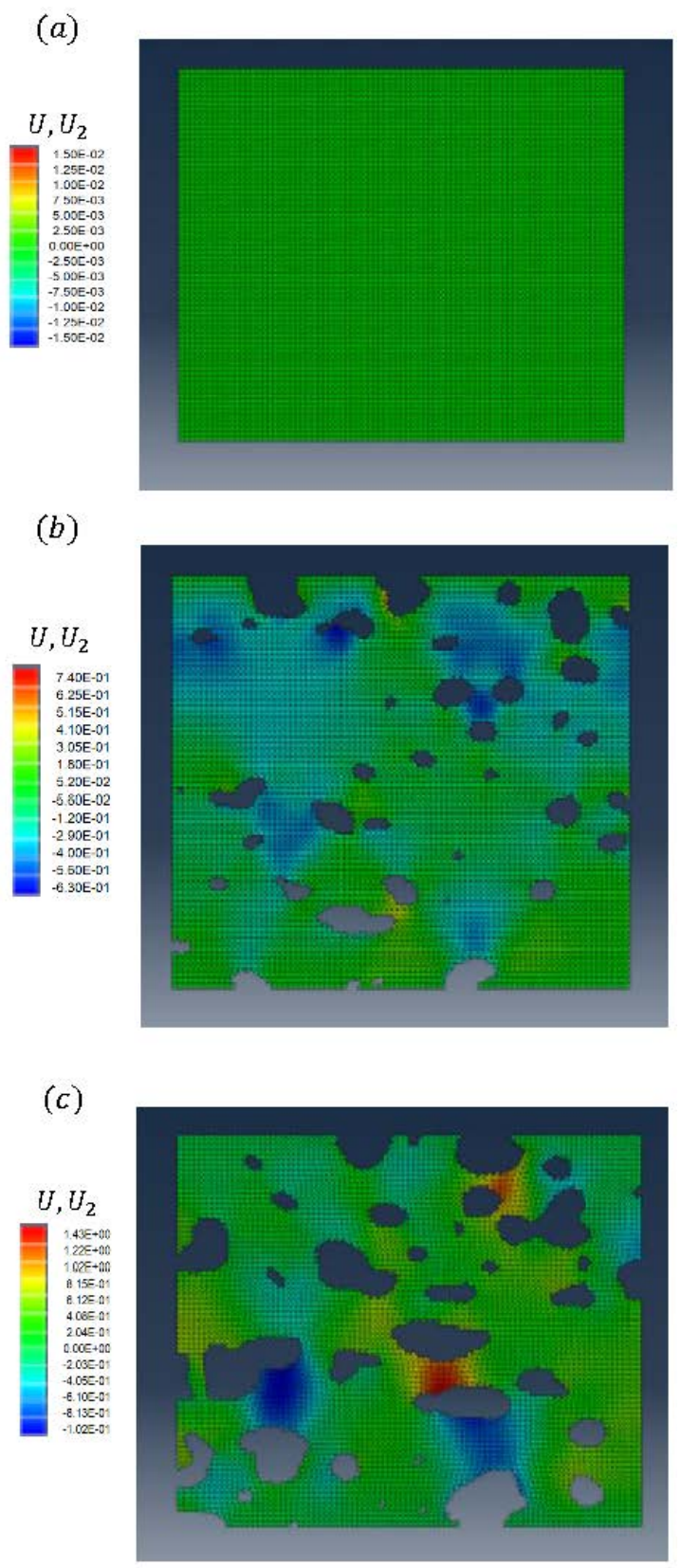

Fig. 3. Deformation perpendicular to the direction of subjected load with: (a) no porosity; (b) $10 \%$ porosity; (c) $20 \%$ porosity. 

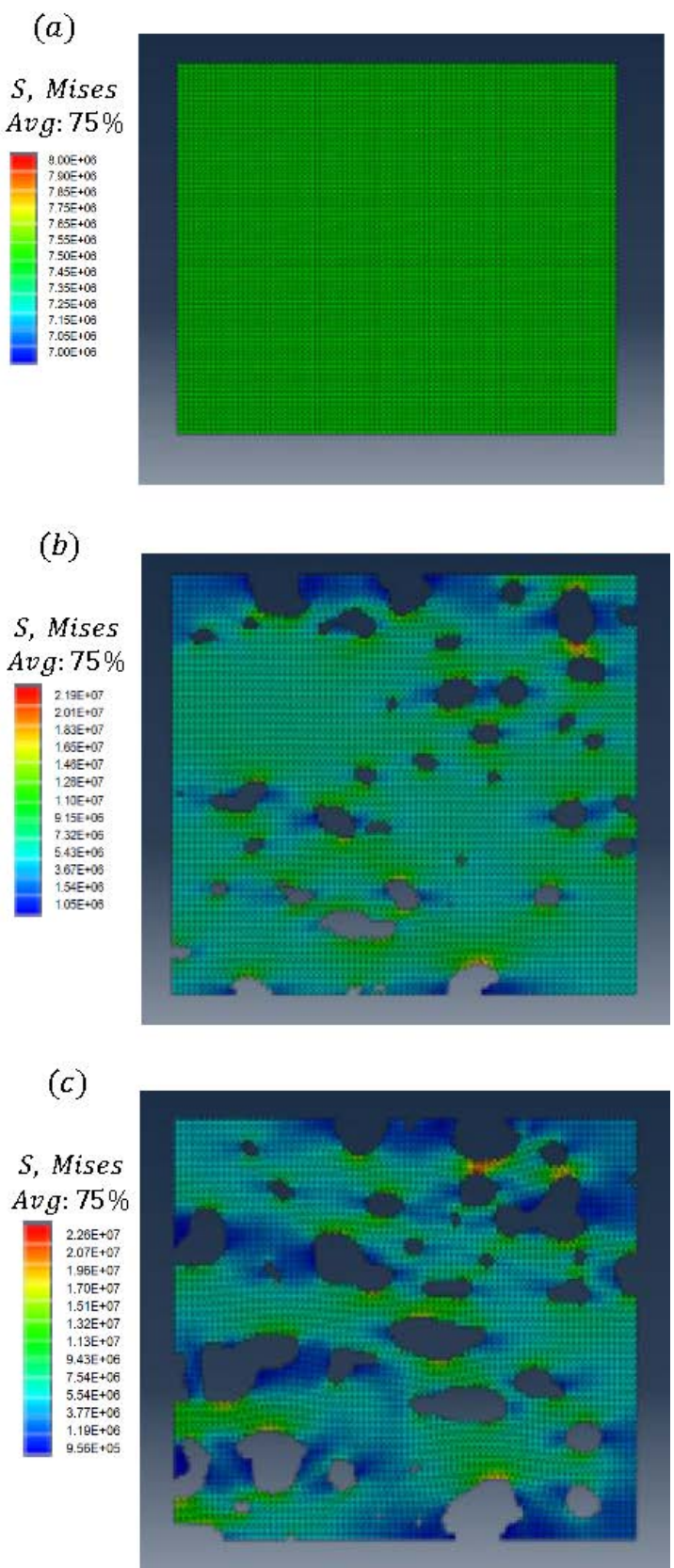

Fig. 4. Von-Mises stress of media with: (a) no porosity; (b) 10\% porosity; (c) $20 \%$ porosity. 

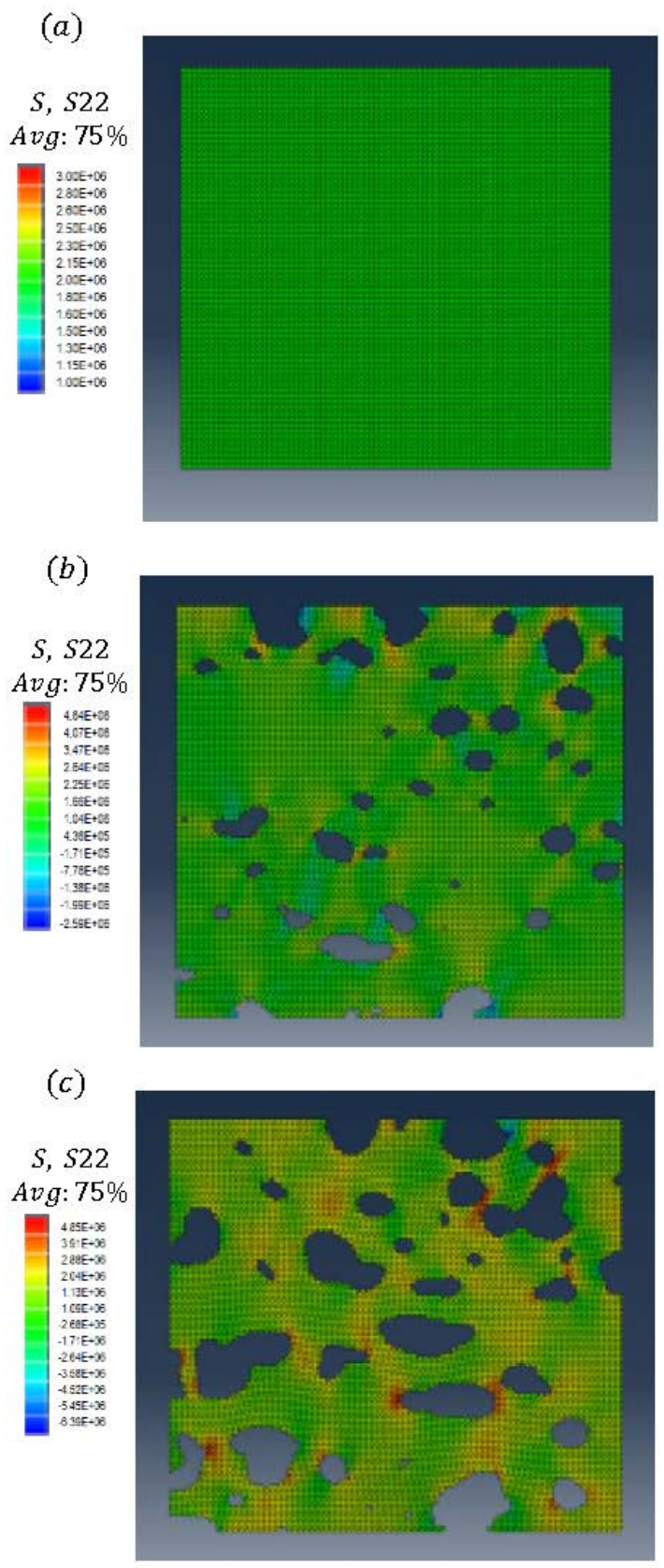

Fig. 5. Stress perpendicular to the direction of subjected load with: (a) no porosity; (b) 10\% porosity; (c) $20 \%$ porosity. 


\section{Conclusion}

This study simulates the porosity of the polymeric networks and performs their structural analysis using nonlinear finite element method. It is concluded that the porosity has remarkable effect on the maximum von-Mises stress value and therefore on the mechanical behavior of the polymeric networks. It is worth noting that two remarks should be considered with respect to capability and validity of the suggested framework. First, the polymer network is supposed to be polydisperse and chains length distribution conforms to simple exponential distribution. Second, it is assumed that the deformations are affine since all the polymer strands undergo same deformation at each moment. Since there is no similar experimental study for comparison, as a final note, this study emphasizes the significance of further investigations for polymers with nano-porosity, which could be a potential topic for future studies.

\section{REFERENCES}

[1] V. Karageorgiou, D. Kaplan (2005) Porosity of 3D biomaterial scaffolds and osteogenesis. Biomaterials 26(27) 5474-5491.

[2] D. Wu, F. Xu, B. Sun, R. Fu, H. He, K. Matyjaszewski (2012) Design and Preparation of Porous Polymers. Chemical Reviews 112(7) 3959-4015.

[3] N. Chaoui, M. Trunk, R. Dawson, J. Schmidt, A. Thomas (2017) Trends \& challenges for microporous polymers. Chemical Society Reviews 46(11) 3302-3321.

[4] N. Enjamuri, S. Sarkar, B.M. Reddy, J. Mondal (2018) Design and Catalytic Application of Functional Porous Organic Polymers: Opportunities and Challenges. Chemical Record Sept.

[5] J.-X. Jiang, Y. Li, X. Wu, J. Xiao, D.J. Adams, A.I. Cooper (2013) Conjugated Microporous Polymers with Rose Bengal Dye for Highly Efficient Heterogeneous Organo-Photocatalysis. Macromolecules 46(22) 8779-8783.

[6] J. Fu, S. Das, G. XING, T. Ben, V. ValtcheV, S. QIU (2016) Fabrication of COFMOF Composite Membranes and Their Highly Selective Separation of H2/CO2. Journal of the American Chemical Society 138(24) 7673-7680.

[7] M. Mohseni, P.S. AbDar, S.M. Borghei (2016) The highest inhibition coefficient of phenol biodegradation using an acclimated mixed culture. Water Science and Technology 73(5) 1033-1040.

[8] F. Xu, X. Chen, Z. TAng, D. Wu, R. Fu, D. Jiang (2014) Redox-active conjugated microporous polymers: a new organic platform for highly efficient energy storage. Chemical Communications 50(37) 4788-4790.

[9] Q. FANG et al. (2015) 3D Porous Crystalline Polyimide Covalent Organic Frameworks for Drug Delivery. Journal of the American Chemical Society 137(26) 8352-8355.

[10] H. DAL, M. KALISKE (2009) A micro-continuum-mechanical material model for failure of rubber-like materials: Application to ageing-induced fracturing. Journal of the Mechanics and Physics of Solids 57(8) 1340-1356. 
[11] M. Tehrani, M.H. Moshaei, A.S. Sarvestani (2017) Network Polydispersity and Deformation-Induced Damage in Filled Elastomers. Macromolecular Theory and Simulations 26(6) 1700038.

[12] H. JinNo ET AL. (2017) Stretchable and waterproof elastomer-coated organic photovoltaics for washable electronic textile applications. Nature Energy 2(10) 780.

[13] P. SKalsKi, K. Kalita (2017) Role of Magnetorheological Fluids and Elastomers in Today's World. Acta Mechanica et Automatica 11(4) 267-274.

[14] J. YAng, A.R. WebB, G.A. Ameer (2004) Novel Citric Acid-Based Biodegradable Elastomers for Tissue Engineering. Advanced Materials 16(6) 511-516.

[15] B. AMSDEN (2007) Curable, biodegradable elastomers: emerging biomaterials for drug delivery and tissue engineering. Soft Matter 3(11) 1335-1348.

[16] J.L. Ifkovits, K. WU, R.L. MAUCK, J.A. BURdick (2010) The Influence of Fibrous Elastomer Structure and Porosity on Matrix Organization. PLOS ONE 5(12) e15717.

[17] M. Tehrani, A. SARVESTANi (2017) Effect of chain length distribution on mechanical behavior of polymeric networks. European Polymer Journal 87 136-146.

[18] M. Tehrani, Z. Ghalamzan, A. Sarvestani (2018) Polydispersity controls the strength of semi-flexible polymer networks. Physical Biology, 15(6) 066002.

[19] D.G. Barrett, W. Luo, M.N. Yousaf (2015) Developing chemoselective and biodegradable polyester elastomers for bioscaffold application. Journal of Materials Chemistry B 3(7) 1405-1414.

[20] M. Tehrani, M.H. Moshaei, A. Sarvestani (2017) Cell mechano-transduction is mediated by receptor diffusion. In: 54th ann. technical meeting society of eng. science 2(34771.89124). https://doi.org/10.13140/rg.

[21] M. Tehrani, M.H. Moshaei, M. Jafari, M. Khalili (2018) On Mechanical Behavior of Elastomeric Networks: Effects of Random Porous Micro-structure. arXiv preprint arXiv:1808.04688.

[22] R. Avazmohammadi, R. Naghdabadi (2013) Effective behavior of porous elastomers containing aligned spheroidal voids. Acta Mechanica 224(9) 1901-1915.

[23] N.S. BakhValov, G. Panasenko (1989) "Homogenisation: Averaging Processes in Periodic Media: Mathematical Problems in the Mechanics of Composite Materials". Springer, Netherlands.

[24] Y.M. Shabana, G.-T. WANG (2013) Thermomechanical modeling of polymer nanocomposites by the asymptotic homogenization method. Acta Mechanica 224(6) 1213-1224.

[25] E. Bosco, R.H.J. Peerlings, M.G.D. Geers (2017) Asymptotic homogenization of hygro-thermo-mechanical properties of fibrous networks. International Journal of Solids and Structures 115-116 180-189.

[26] S.S. Vel, A.J. Goupee (2010) Multiscale thermoelastic analysis of random heterogeneous materials: Part I: Microstructure characterization and homogenization of material properties. Computational Materials Science 48(1) 22-38. 
[27] E.M. ARrudA, M.C. BoyCe (1993) A three-dimensional constitutive model for the large stretch behavior of rubber elastic materials. Journal of the Mechanics and Physics of Solids 41(2) 389-412.

[28] M.J. Tehrani (2017) "Micromechanical Analysis of Strength of Polymer Networks with Polydisperse Structures". Thesis, Faculty of the Russ College of Engineering and Technology of Ohio University.

[29] M. Tehrani, M.H. Moshaei, A. Sarvestani (2017) "Revisiting the DeformationInduced Damage in Filled Elastomers: Effect of Network Polydispersity," arXiv:1710.01846 [cond-mat, physics:physics].

[30] W. Sun, E.L. Chaikof, M.E. Levenston (2008) Numerical Approximation of Tangent Moduli for Finite Element Implementations of Nonlinear Hyperelastic Material Models. Journal of Biomechanical Engineering 130(6) 061003.

[31] K.-J. BAthe (2014) "Finite Element Procedures, Second edition. S.l.

[32] C. Miehe (1996) Numerical computation of algorithmic (consistent) tangent moduli in large-strain computational inelasticity. Computer Methods in Applied Mechanics and Engineering 134(3) 223-240.

[33] M. SHAHZAD et al. (2015) Mechanical Characterization and FE Modelling of a Hyperelastic Material. Materials Research 18(5) 918-924. 